\title{
Perfil do paciente obeso e portador de síndrome metabólica candidato à cirurgia bariátrica em uma clínica particular de Porto Alegre, Rio Grande do Sul
}

\author{
Profile of obese patients with metabolic syndrome eligible for bariatric surgery \\ at a private clinic in Porto Alegre, Rio Grande do Sul, Brazil
}

\author{
Vilma Maria Junges ${ }^{1}$, Jarbas Marinho Branco Cavalheiro², Eliana Franzoi Fam², Vera Elizabeth Closs ${ }^{1}$, \\ Maria Gabriela Valle Gottlieb ${ }^{1} \bowtie$ \\ ${ }^{1}$ Programa de Pós-graduação em Geriatria e Gerontologia na Pontifícia Universidade Católica do Rio Grande do Sul (PUCRS). Porto Alegre, RS, Brasil. \\ ${ }^{2}$ Centro Integrado de Tratamento da Obesidade (CINTRO). Porto Alegre, RS, Brasil.
}

\section{RESUMO}

Objetivos: Descrever o perfil sociodemográfico, de comorbidades, bioquímico, de estilo de vida e de uso de medicamentos de pacientes obesos, portadores de síndrome metabólica, candidatos à cirurgia bariátrica.

Métodos: Este estudo transversal retrospectivo incluiu indivíduos obesos grau I, II e III, portadores de síndrome metabólica e candidatos à cirurgia bariátrica, atendidos em uma clínica para tratamento da obesidade, em Porto Alegre, Rio Grande do Sul. Os pacientes foram atendidos entre janeiro de 2007 e março de 2014 e os dados para estudo foram coletados dos prontuários médicos. As variáveis investigadas foram: idade, sexo, escolaridade, estado civil, comorbidades, marcadores bioquímicos, uso de medicamentos, histórico de tabagismo, história de excesso de peso na infância e qualidade do sono. Os pacientes foram classificados com obesidade grau I (IMC=30-34,9), obesidade grau II $(\mathrm{IMC}=35-39,9)$ e obesidade grau III, ou grave (IMC $\geq 40)$. O diagnóstico de síndrome metabólica foi realizado de acordo com a International Diabetes Federation.

Resultados: Foram avaliados 198 indivíduos, com média de idade de 40,5 $\pm 11,1$ anos (intervalo de 21 a 69 anos), dos quais $83,8 \%$ eram mulheres e $68,7 \%$ eram casados. A faixa etária mais frequente foi de 30 a 39 anos $(37,4 \%)$. Com relação às condições de saúde, $77,3 \%$ dos pacientes tinham hipertensão arterial sistêmica, 75,8\% eram dislipidêmicos e 75,3\% eram portadores de gastrite, destacando-se que $67,2 \%$ dos pacientes utilizavam medicamentos anti-hipertensivos e $42,9 \%$ faziam uso de medicação psiquiátrica. Com relação aos níveis de proteína C reativa, verificou-se uma mediana de $0,81(0,70-0,97 \mathrm{mg} / \mathrm{dL})$. Cento e treze pacientes $(57 \%)$ referiam ter tido peso excessivo na infância. Má qualidade do sono foi mencionada por $61(31 \%)$ dos indivíduos.

Conclusões: A maior parte dos indivíduos com obesidade grau I, II e III e síndrome metabólica, que procurou atendimento na clínica de cirurgia bariátrica, era constituída de mulheres adultas jovens, casadas, com ensino de nível superior ou especializado e com a presença de várias comorbidades somadas ao excesso de peso e caráter inflamatório. Outros achados frequentes incluíram alterações do sono, relato de peso excessivo na infância e uso de mais de um tipo de medicamento.

DESCRITORES: obesidade; perfil de saúde; síndrome X metabólica; uso de medicamentos; cirurgia bariátrica.

\section{ABSTRACT}

Aims: To describe the sociodemographic and biochemical profiles, presence of comorbidities, lifestyles, and drug utilization of obese patients with metabolic syndrome eligible for bariatric surgery.

Methods: This retrospective cross-sectional study included metabolic syndrome patients with class I, II, and III obesity treated at a specialized clinic in Porto Alegre, Rio Grande do Sul, Brazil, who were candidates for bariatric surgery. The patients were seen between January 2007 and March 2014 and the data for the study were collected from medical records. The following variables were investigated: age, sex, education, marital status, comorbidities, biochemical markers, drug utilization, smoking history, history of childhood overweight, and sleep quality. Patients were classified as class I obesity $(\mathrm{BMI}=30$ to 34.9$)$, class II obesity (BMI=35 to 39.9), and class III, or severe, obesity $(\mathrm{BMI} \geq 40)$. The diagnosis of metabolic syndrome was established according to the International Diabetes Federation.

Results: A total of 198 subjects $(83.8 \%$ of whom were women and $68.7 \%$ were married) with a mean age of $40.5 \pm 11.1$ years (range of $21-69$ years) were evaluated. Most of the patients (37.4\%) were 30 to 39 years old. With regard to health, $77.3 \%$ of the patients had hypertension, $75.8 \%$ had dyslipidemia, and $75.3 \%$ had gastritis, and it should be noted that $67.2 \%$ of the patients were on antihypertensive medications and $42.9 \%$ on psychiatric drugs. A median of $0.81(0.70-0.97 \mathrm{mg} / \mathrm{dL})$ was obtained for C-reactive protein levels. One hundred and thirteen patients $(57 \%)$ reported having had excessive weight in childhood. Poor sleep quality was mentioned by 61 (31\%) of individuals.

Conclusions: Most individuals with class I, II, and III obesity and metabolic syndrome who sought care at the bariatric surgery clinic were young, adult, and married women with a college degree or higher education presenting with several comorbidities in addition to excess weight and inflammatory conditions. Other common findings included sleep disorders, excessive weight in childhood, and use of more than one type of medication.

KEY WORDS: obesity; health profile; metabolic syndrome X; drug utilization; bariatric surgery. 
Abreviaturas: ATC, Anatomical Therapeutic Chemical; CINTRO, Centro Integrado de Tratamento da Obesidade; DCV, doenças cardiovasculares; DM2, diabetes mellitus tipo 2; HAS, hipertensão arterial sistêmica; HDL-c, colesterol ligado à lipoproteína de alta densidade; IDF, International Diabetes Federation; IMC, índice de massa corporal; LDL-c, colesterol ligado à lipoproteína de baixa densidade; NASH, doença hepática não alcoólica; OMS, Organização Mundial da Saúde; PAD, pressão arterial diastólica; PAS, pressão arterial sistólica; PCR, proteína $\mathrm{C}$ reativa; $\mathrm{SM}$, síndrome metabólica; $\mathrm{TSH}$, hormônio estimulante da tireoide.

\section{INTRODUÇÃO}

A obesidade, de acordo com a Organização Mundial da Saúde (OMS), é um estado mórbido caracterizado pelo excesso de acúmulo de gordura corporal conforme o índice de massa corporal (IMC). A obesidade está associada a maior risco de diabetes mellitus do tipo 2 (DM2), doenças cardiovasculares (DCV), alguns tipos de câncer, aumento da morbidade e mortalidade, maiores gastos com a saúde e, consequentemente, redução da expectativa de vida $[1,2]$.

Um percentual dos indivíduos obesos apresenta também um conjunto de fatores de risco cardiometabólicos caracterizados como síndrome metabólica (SM). A SM é definida pela presença de DM2 (alterações no metabolismo dos carboidratos como intolerância à glicose, hiperinsulinemia e resistência à insulina), hipertensão arterial sistêmica (HAS), obesidade abdominal e dislipidemia [3]. A dislipidemia inclui alterações no metabolismo das gorduras como diminuição do colesterol ligado à lipoproteína de alta densidade (HDL-c) e elevação dos níveis de triglicerídeos. Estudos também têm demonstrado que indivíduos portadores de SM apresentam um estado pró-inflamatório, em que os níveis de proteína $\mathrm{C}$ reativa (PCR), interleucina 6 e fator de necrose tumoral alfa estão elevados [3]. Além disso, a SM está associada a uma maior mortalidade por DCV em torno de duas vezes e meia, e a maior mortalidade geral em aproximadamente uma vez e meia. A obesidade, a predisposição genética, a alimentação inadequada e o sedentarismo estão entre os fatores de risco mais importantes e decisivos para o aparecimento dessa síndrome [4].

Estudos têm demonstrado, também, que os tratamentos convencionais que contemplam farmacoterapia, adesão a uma dieta equilibrada e saudável e prática regular de exercícios físicos para a redução de peso e dos componentes da SM são muito benéficos e apresentam resultados positivos. Entretanto, para os indivíduos obesos grau II e III, que são portadores de
SM, esses tratamentos convencionais não apresentam a eficácia esperada, havendo pouco sucesso em longo prazo, principalmente devido à grande dificuldade de o paciente aderir ao tratamento [5].

Apesar das políticas públicas implantadas no Brasil para inibir a evolução da obesidade para obesidade grave (definida por um IMC $\geq 40 \mathrm{~kg} / \mathrm{m}^{2}$ ), ainda existe no país um número muito expressivo de indivíduos com esse grau de obesidade [6]. A obesidade grave é refratária à terapia com dieta alimentar e medicamentos, mas tem tido uma boa resposta à cirurgia bariátrica [7] Por esse motivo, a cirurgia bariátrica tem sido indicada como tratamento para a perda de peso e também para o controle de DM2, HAS e dislipidemias, que, em conjunto, ocorrem na SM. Evidências têm mostrado que a cirurgia bariátrica é o tratamento mais eficaz para as comorbidades associadas à obesidade grave [5], resultando em redução da mortalidade total em 30\% e redução significativa na ocorrência de câncer [2].

Embora as publicações sobre cirurgia bariátrica existam em grande volume na literatura internacional, há poucos estudos no Brasil sobre o perfil do paciente candidato a esse tipo de cirurgia. $\mathrm{O}$ aparecimento de algumas comorbidades é indicativo de complicações e até mesmo óbito em indivíduos submetidos à cirurgia bariátrica. Assim, conhecer o perfil dos pacientes é de grande valia para avaliar os riscos de morbimortalidade da cirurgia e provisionar recursos técnicos. O objetivo deste trabalho foi descrever o perfil sociodemográfico, de comorbidades, bioquímico, de estilo de vida e de uso de medicamentos de paciente obesos, portadores de SM, com indicação de cirurgia bariátrica.

\section{MÉTODOS}

Este estudo transversal retrospectivo foi realizado a partir da revisão de prontuários médicos dos pacientes. A população investigada foi constituída por pacientes encaminhados ao Centro Integrado de Tratamento da Obesidade (CINTRO), localizado em Porto Alegre, Rio Grande do Sul. São pacientes que consultam em caráter privativo ou por planos de saúde, principalmente por indicação de cardiologistas, endocrinologistas e ortopedistas, mas também que chegam ao CINTRO por meio de informações da mídia ou de outros pacientes que já realizaram procedimento cirúrgico nessa clínica.

A amostra foi composta por pacientes obesos grau I, II e III, portadores de SM e que tiveram indicação de cirurgia bariátrica. A anamnese pré-cirúrgica foi realizada por uma equipe multiprofissional (médicos, nutricionista, psicóloga e educadora física) no período de janeiro de 2007 a março de 2014. Os critérios de 
inclusão para este estudo foram: idade mínima de 20 anos, ser portador de obesidade e SM e ter a indicação de cirurgia bariátrica. Foram revisados 273 prontuários de pacientes, sendo que 198 destes foram considerados elegíveis, pois preencheram os critérios de inclusão.

As informações sobre condições sociodemográficas, uso de medicamentos, estilo de vida, histórico de tabagismo, excesso de peso na infância, transtorno de comer compulsivo, bulimia e qualidade do sono foram obtidas por autorrelato dos pacientes e estavam registradas nos prontuários. Foram obtidas também informações sobre uso de medicamentos para HAS, diabetes ou dislipidemia. A anamnese foi realizada pela equipe multiprofissional do CINTRO no momento da avaliação pré-cirúrgica.

Informações sobre variáveis antropométricas, clínicas e bioquímicas foram obtidas a partir de análises laboratoriais e dos exames objetivos realizados na clínica e também anotados nos prontuários. As variáveis antropométricas foram estatura, aferida com estadiômetro com balança; peso, aferido com balança antropométrica (balança Toledo ${ }^{\circledR}$ antropométrica com régua, $200 \mathrm{~kg}$ ); IMC, calculado por meio da equação de Quetelet $\left(\mathrm{IMC}=\right.$ peso/altura $\left.{ }^{2}\right)$ [10]; e circunferência abdominal, medida no ponto médio entre a crista ilíaca e o rebordo costal.

A obesidade foi classificada da seguinte forma: IMC $=30-34,9-$ obesidade grau I; IMC $=35-39,9-$ obesidade grau II; e IMC $\geq 40$ - obesidade grave ou mórbida [11]. Já valores de pressão arterial até 130/ $85 \mathrm{mmHg}$ foram considerados normais, de acordo com as recomendações da International Diabetes Federation (IDF) [4]. A pressão arterial foi aferida com auxílio de um esfigmomanômetro aneroide e estava anotada no prontuário do paciente.

Os exames bioquímicos foram coletados na avaliação pré-cirúrgica: glicemia de jejum, triglicerídeos, colesterol total, HDL-c, colesterol ligado à lipoproteína de baixa densidade (LDL-c), hormônio estimulador da tireoide (TSH), PCR, folato, vitamina B12 e ferro.

O diagnóstico de SM foi realizado pelos autores, a partir dos dados dos prontuários, de acordo com o critério do IDF, que define um indivíduo como portador de SM quando apresentar obesidade central e dois dos seguintes critérios: triglicerídeos $\geq 150 \mathrm{mg} /$ $\mathrm{dL}$ ou tratamento para dislipidemia; HDL-c $<40 \mathrm{mg} / \mathrm{dL}$ em homens ou $<50 \mathrm{mg} / \mathrm{dL}$ em mulheres ou tratamento para dislipidemia; pressão arterial sistólica (PAS) $\geq 130 \mathrm{mmHg}$ ou pressão arterial diastólica $\geq 85 \mathrm{mmHg}$ ou diagnóstico prévio de HAS; e glicemia de jejum $\geq 100 \mathrm{mg} / \mathrm{dL}$ ou diagnóstico prévio de DM2 ou uso de hipoglicemiante. Como previsto no consenso do
IDF, quando não havia a mensuração da circunferência abdominal, os indivíduos que apresentavam IMC $>30 \mathrm{~kg} / \mathrm{m}^{2}$, foram considerados com critério positivo para o diagnóstico da SM no que se refere à obesidade central [4].

O diagnóstico de esteatose hepática foi obtido através de exames de imagem, como ultranossografia abdominal total e/ou tomografia computadorizada e/ou ressonância nuclear magnética, bem como por meio de biópsia hepática. $\mathrm{O}$ diagnóstico de gastrite foi feito pelo exame de endoscopia gástrica, que é solicitado de rotina para todos os pacientes da clínica.

Os medicamentos em uso pelos pacientes foram classificados pelo sistema Anatomical Therapeutic Chemical (ATC), que divide os princípios ativos em diferentes grupos terapêuticos de acordo com seus locais de ação e suas características farmacológicas e químicas. Para tanto, foi utilizada a revisão de 2000 e atualizações. Na ATC, os fármacos são classificados de acordo com seu uso terapêutico mais importante [9].

Os dados foram armazenados em planilha do Excel e analisados pelo pacote estatístico IBM SPSS 17.0. Os dados contínuos foram previamente testados para normalidade pelo teste de Kolmogorov-Smirnov. As variáveis categóricas foram descritas por meio de frequências (absolutas e relativas), e as variáveis contínuas por meio de medidas de tendência central: média, desvio-padrão, mediana e intervalo interquartil (IQ).

O estudo foi aprovado pelo Comitê de Ética em Pesquisa da Pontifícia Universidade Católica do Rio Grande do Sul (PUCRS), sob o parecer consubstanciado de número 506.428. Foi colhida assinatura do pesquisador em termo de confidencialidade e autorização do coordenador do CINTRO para a utilização das informações para a pesquisa, sendo dispensada a assinatura de consentimento informado por parte dos pacientes por tratar-se de estudo retrospectivo.

\section{RESULTADOS}

Foram avaliados 198 indivíduos obesos e portadores de SM, com mediana de idade de 32 anos (IQ 38-50, mínima 21, máxima 69 anos), sendo a maioria da amostra constituída de mulheres $(83,8 \%)$. Quanto à escolaridade, a maioria $(93,0 \%)$ dividia-se, igualmente, em graduados em curso superior $(46,5 \%)$ e graduados com curso de especialização (46,5\%) (Tabela 1).

O critério de diagnóstico de SM mais frequente foi a obesidade central, seguido dos triglicerídeos aumentados e da HAS (Tabela 2). 
Em relação ao uso de medicamentos, a amostra consumia uma mediana de dois medicamentos (intervalo interquartil de 1-4) no pré-cirúrgico, sendo que $67,7 \%$ dos indivíduos faziam uso de mais de um medicamento diário. Os tipos de medicamentos utilizados, as condições de saúde dos pacientes, a autopercepção corporal na infância, a qualidade do sono, o histórico de tabagismo, os dados antropométricos e o estado nutricional antes da cirurgia estão descritos na Tabela 3.

Tabela 1. Perfil sociodemográfico da amostra de pacientes obesos e portadores de síndrome metabólica candidatos à cirurgia bariátrica.

\begin{tabular}{lc}
\hline \multicolumn{1}{c}{ Variáveis } & $\mathbf{n}(\%)$ \\
Idade em anos - mediana (IQ) & $38(32-50)$ \\
Faixa etária (anos) & \\
$20-29$ & $33(16,7)$ \\
$30-39$ & $74(37,4)$ \\
$40-49$ & $41(20,7)$ \\
50 ou mais & $50(25,3)$ \\
Sexo feminino & $166(83,8)$ \\
Estado civil & \\
Casado & $136(68,7)$ \\
Solteiro & $44(22,2)$ \\
Divorciado & $15(7,6)$ \\
Viúvo & $3(1,5)$ \\
Escolaridade & \\
Segundo grau & $2(1,0)$ \\
Superior & $92(46,5)$ \\
Especialização & $92(46,5)$ \\
Mestrado/doutorado & $11(5,6)$ \\
Curso técnico & $1(0,5)$ \\
\hline
\end{tabular}

$\mathrm{IQ}$, intervalo interquartil.

Tabela 2. Frequência dos critérios para diagnóstico de síndrome metabólica da amostra de pacientes obesos e portadores de síndrome metabólica candidatos à cirurgia bariátrica.

\begin{tabular}{lcc}
\hline \multicolumn{1}{c}{ Critérios para síndrome metabólica } & $\begin{array}{c}\text { Amostra } \\
\text { total* }\end{array}$ & n (\%) \\
Obesidade central & 198 & $198(100,0)$ \\
IMC $>30 \mathrm{~kg} / \mathrm{m}^{2}$ & 137 & $137(100,0)$ \\
Circunferência abdominal aumentada & 195 & $180(92,3)$ \\
Triglicerídeos aumentados & 178 & $161(90,4)$ \\
Pressão arterial aumentada & 163 & $95(58,3)$ \\
HDL-c reduzido & 195 & $107(54,9)$ \\
Glicemia de jejum aumentada & & \\
\hline
\end{tabular}

* As diferenças no número de indivíduos na amostra total de cada variável correspondem a dados faltantes para alguns pacientes.

HDL-c, colesterol ligado a lipoproteína de alta densidade.

IMC, índice de massa corporal.
Tabela 3. Frequência de comorbidades, uso de medicamentos, dados antropométricos, histórico de excesso de peso na infância, tabagismo e qualidade do sono de pacientes obesos e portadores síndrome metabólica candidatos à cirurgia bariátrica.

\begin{tabular}{|c|c|c|}
\hline Variáveis & $\begin{array}{c}\text { Amostra } \\
\text { total }\end{array}$ & n (\%) \\
\hline \multicolumn{3}{|l|}{ Comorbidades } \\
\hline Diabetes mellitus & 198 & $52(26,3)$ \\
\hline Dislipidemia & 198 & $150(75,8)$ \\
\hline Hipertensão arterial sistêmica & 198 & $153(77,3)$ \\
\hline Gastrite & 198 & $149(75,3)$ \\
\hline Esteatose hepática & 197 & $137(69,5)$ \\
\hline $\begin{array}{l}\text { Histórico de transtorno do comer } \\
\text { compulsivo }\end{array}$ & 197 & $83(42,1)$ \\
\hline Histórico de bulimia & 197 & $11(5,6)$ \\
\hline \multicolumn{3}{|l|}{ Uso de medicamentos } \\
\hline Hipoglicemiante & 198 & $32(16,2)$ \\
\hline Anticolestorolêmicos & 198 & $21(10,6)$ \\
\hline Anti-hipertensivos & 198 & $133(67,2)$ \\
\hline Gastroprotetores & 198 & $19(9,6)$ \\
\hline Psiquiátricos & 198 & $85(42,9)$ \\
\hline Diuréticos & 198 & $35(17,7)$ \\
\hline Tiroidianos & 198 & $25(12,6)$ \\
\hline Cardíacos & 198 & $6(3,0)$ \\
\hline Anti-inflamatórios & 198 & $7(3,5)$ \\
\hline Anticoagulantes & 198 & $5(2,5)$ \\
\hline Antiartrose & 198 & $3(1,5)$ \\
\hline Antiasmáticos & 198 & $7(3,5)$ \\
\hline Emagrecedores & 198 & $8(4,0)$ \\
\hline Antiflatulência & 198 & $1(0,5)$ \\
\hline \multicolumn{3}{|l|}{$\begin{array}{l}\text { Autorrelato de autopercepção corporal } \\
\text { na infância }\end{array}$} \\
\hline Magro & & $43(21,8)$ \\
\hline Eutrófico (“normal”) & 107 & $41(20,8)$ \\
\hline Excesso de peso & & $78(39,6)$ \\
\hline Grande excesso de peso ("obeso") & & $35(17,8)$ \\
\hline \multicolumn{3}{|l|}{ Qualidade do sono } \\
\hline Boa & 19 & $136(69,0)$ \\
\hline Ruim & & $61(31,0)$ \\
\hline Histórico de tabagismo & 197 & $18(9,1)$ \\
\hline Peso pré-cirurgia em kg (média $\pm D P$ ) & 198 & $114,5 \pm 20,8$ \\
\hline IMC pré-cirurgia em kg/m² (média $\pm D P$ ) & 198 & $42,1 \pm 5,6$ \\
\hline \multicolumn{3}{|l|}{ Estado nutricional pré-cirurgia } \\
\hline Obesidade grau I & & $9(4,5)$ \\
\hline Obesidade grau II & 198 & $64(32,3)$ \\
\hline Obesidade grau III (severa, ou mórbida) & & $125(63,1)$ \\
\hline
\end{tabular}


Tabela 4. Perfil bioquímico e níveis pressóricos de pacientes obesos e portadores de síndrome metabólica candidatos à cirurgia bariátrica.

\begin{tabular}{lccc}
\hline \multicolumn{1}{c}{ Variáveis } & Amostra total* & Média \pm DP & Mediana (IQ) \\
\hline Pressão arterial sistólica $(\mathrm{mmHg})$ & 120 & - & $140,0(120,0-150,0)$ \\
Pressão arterial diastólica $(\mathrm{mmHg})$ & 120 & - & $82,5(80,0-100,0)$ \\
Glicemia de jejum (mg/dL) & 195 & - & $99,0(90,0-108,0)$ \\
Triglicerídeos (mg/dL) & 168 & $172,5 \pm 85,0$ & - \\
Colesterol total (mg/dL) & 169 & $202,1 \pm 38,1$ & - \\
LDL-colesterol (mg/dL) & 43 & $105,4 \pm 38,9$ & - \\
HDL-colesterol $(\mathrm{mg} / \mathrm{dL})$ & 163 & $47,3 \pm 12,1$ & - \\
Homens & 29 & $40,2 \pm 10,4$ & - \\
Mulheres & 185 & $48,6 \pm 12,0$ & - \\
Hormônio estimulante da tireoide (mIU/L) & 164 & - & $2,68(1,80-3,79)$ \\
Proteína C reativa $(\mathrm{mg} / \mathrm{dL})$ & 154 & - & $0,81(0,70-0,97)$ \\
Folato $(\mathrm{ng} / \mathrm{mL})$ & 133 & $11,2 \pm 8,5$ & - \\
Vitamina B12 $(\mathrm{pg} / \mathrm{mL})$ & 144 & - & $383,5(302,3-530,0)$ \\
Ferro $(\mu \mathrm{g} / \mathrm{dL})$ & 131 & $86,6 \pm 25,2$ & - \\
\hline
\end{tabular}

* Algumas variáveis têm dados faltantes.

DP, desvio padrão; IQ, intervalo interquartil; LDL, lipoproteína de baixa densidade; HDL, lipoproteína de alta densidade.

Na Tabela 4 observa-se que, conforme os critérios da IDF, a PAS teve mediana acima do considerado adequado $(140 \mathrm{mmHg})$ caracterizando HAS [4]. A mediana da glicemia de jejum $(99 \mathrm{mg} / \mathrm{dL})$ ficou em níveis normais, mas limítrofe. Em relação perfil lipídico pode-se verificar que em média apresentaram valores acima (triglicerídeos, colesterol e LDL) ou abaixo dos valores de referência, como por exemplo a média do HDL-c, que foi de 40,2 $\pm 10,4 \mathrm{mg} / \mathrm{dL}$ para os homens e $48,6 \pm 12,0 \mathrm{mg} / \mathrm{dL}$ para as mulheres (valor considerado baixo para mulheres).

Os valores da mediana do TSH $(2,68 \mathrm{mIU} / \mathrm{L})$, ácido fólico $(11,2 \mathrm{ng} / \mathrm{mL})$, vitamina B12 $(383 \mathrm{pg} / \mathrm{mL})$, bem como dos níveis de ferro $(86,6 \pm 25,2 \mu \mathrm{g} / \mathrm{dL})$ estavam dentro dos valores de referência considerados normais. Contudo, a mediana da PCR encontrada $(0,81 \mathrm{mg} / \mathrm{dL})$ estava acima dos valores de referência (Tabela 4).

\section{DISCUSSÃO}

O objetivo deste estudo foi descrever o perfil sociodemográfico, de comorbidades, bioquímico, de estilo de vida e de uso de medicamentos de pacientes obesos, portadores de síndrome metabólica, candidatos à cirurgia bariátrica. Os resultados mostraram que a amostra investigada apresenta um perfil predominantemente feminino e de casados. Este dado parece indicar que há uma procura maior desse tipo de tratamento pelas mulheres, sendo semelhante ao encontrado em outros estudos [10,11]. Estudos indicam também que o maior contingente de pessoas casadas pode ser consequência da necessidade de apoio por parte do cônjuge e da família em um momento de grandes mudanças pessoais, de saúde, sociais, físicas e emocionais pelo qual passam os pacientes obesos que realizam cirurgia bariátrica $[12,13]$.

Quanto à faixa etária, o grupo com idade entre 30 e 39 anos foi predominante, seguido pela faixa etária acima de 50 anos. Ressalta-se que o tempo de obesidade na vida do paciente dificulta mais ainda a perda de peso. O excesso de peso produz distúrbios metabólicos conhecidos, com efeitos severos e nocivos sobre o corpo, e a idade, o tempo de obesidade, a presença de diabetes e o seu tempo de evolução podem acarretar alterações específicas e cumulativas no metabolismo [14]. Ao analisar o grau de escolaridade, percebeu-se a predominância de indivíduos com curso superior e especialização sobre os demais níveis de escolaridade. Esse achado pode estar relacionado ao perfil econômico elevado na população estudada.

Neste estudo, o critério para diagnóstico de SM mais frequente foi a obesidade central, calculada de acordo com o IMC, seguido por triglicerídeos aumentados e pela pressão arterial elevada. Tais resultados evidenciam a profunda relação entre obesidade e fatores de risco para $\mathrm{DCV}$, trazendo à luz um quadro preocupante sobre as condições de saúde do paciente obeso com SM [15].

Além disso, outros achados são preocupantes. Embora os pacientes se mostrassem assintomáticos, determinados resultados de exames solicitados no protocolo de cirurgia bariátrica indicavam problemas 
de saúde ainda não identificados. Por exemplo, na amostra avaliada, $75,3 \%$ dos pacientes apresentavam gastrite, dos quais apenas 9,6\% estavam utilizando medicação gastroprotetora, ou seja, grande parte dos pacientes com gastrite não estava em tratamento. A gastrite é uma doença grave e que tem o potencial para desenvolver câncer de estômago [16]. Ademais, já foi encontrada relação entre gastrite e hiperlipidemia, doença coronária, infarto do miocárdio, terapia de hiperlipidemia e drogas hipotensoras em homens, sugerindo que a gastrite tem potencial associação com DCV nesse segmento da população [16,19]. Grande parte de amostra investigada apresentou também resultado positivo para esteatose hepática, o que indica risco para doença hepática não alcoólica (NASH). $\mathrm{O}$ acúmulo de gordura no fígado é clinicamente importante, porque um número preocupante de pacientes desenvolverá NASH, podendo em seguida progredir para fibrose, cirrose e insuficiência hepática. Esse quadro de obesidade grave somada à esteatose hepática pode piorar o estado de saúde, causando, inclusive, o óbito do paciente [17,18].

Observou-se, também, que mais de $40 \%$ dos pacientes tinham histórico de transtorno de comer compulsivo, e 5,6\% relataram já ter tido bulimia, podendo indicar uma relação entre transtornos alimentares e obesidade. A obesidade diminui a qualidade de vida, sendo agravada pela associação com comorbidades coexistentes, e o transtorno de compulsão alimentar está relacionado com imagem corporal e com excesso de peso [19]. O excesso de peso associado às comorbidades, que são parte do perfil do indivíduo obeso portador de SM, induz ao uso de muitos medicamentos, às vezes de diferentes classes, o que pode acarretar diferentes efeitos e riscos.

O presente estudo também avaliou os medicamentos consumidos pela amostra no período pré-cirúrgico. $\mathrm{O}$ uso de mais de um medicamento pode ocasionar mudanças na ação esperada dos fármacos, devido à interação medicamentosa. Em pacientes críticos, como os candidatos à cirurgia bariátrica, interações medicamentosas são ainda mais preocupantes, pois, submetidos a esquemas terapêuticos múltiplos, esses indivíduos podem sofrer consequências oriundas da falência de sistemas responsáveis pela absorção, metabolização e excreção dos fármacos [20].

Sotolongo et al. [21], em pesquisa realizada em Cuba com pacientes hospitalizados portadores de SM, encontraram associação positiva entre o aumento da prevalência de comorbidades ligadas à $\mathrm{SM}$ e uso concomitante de polifarmácia. Apesar de que o uso de muitos medicamentos foi identificado como um fator que interfere negativamente na morbidade e mortalidade desses pacientes, a conduta preconizada é não interromper o seu uso, pois o tratamento insuficiente é também causador de morte. O tratamento da SM requer a administração de um maior número de medicamentos, o que pode incrementar o perfil de comorbidade desse paciente. Nesse sentido, os autores recomendam a perda de peso e a consequente melhora metabólica, que pode ser alcançada com a cirurgia bariátrica. Após a intervenção cirúrgica, existe a chance de diminuir o uso de medicamentos em classes e doses ou mesmo de eliminar alguns [21].

$\mathrm{O}$ excesso de peso pode ser uma preocupação de longa data na vida do indivíduo obeso, como observado nesta amostra. A maior parte dos indivíduos se autorrelatou como tendo excesso de peso, ou mesmo como obeso, já na infância. Indivíduos com problemas relacionados ao peso desde idades precoces revelam a dificuldade de tratar a obesidade. Além das dificuldades no tratamento da obesidade e dos riscos da SM para DCV, câncer e acidentes vasculares, 9,1\% dos pacientes relataram histórico de tabagismo, e $31 \%$ referiram qualidade de sono ruim. Tais fatores prejudicam, consideravelmente, a qualidade de vida desses indivíduos e aumentam o risco de mortalidade não somente por $\mathrm{DCV}$, mas também de forma geral [22].

O grau de obesidade, segundo o IMC, também pode ser um agravante na condição do paciente com SM, visto que algumas comorbidades agravam-se com o aumento do peso, como a HAS [23]. O IMC médio dos pacientes pesquisados evidencia um grau preocupante de obesidade e possivelmente de agravamento de suas comorbidades. Dos indivíduos avaliados, 125 foram classificados como obesos grau III $(63,1 \%), 64$ como obesos grau II $(32,3 \%)$, e nove $(4,5 \%)$ como obesos grau I, conforme critérios da OMS [1]. O que fica claro acerca da amostra avaliada é que o grupo com obesidade mais severa é o mais numeroso.

A qualidade do sono é importante para todos, entretanto, particularmente nos obesos, sua falta pode ser causa e consequência de comorbidades. Pacientes com apneia obstrutiva do sono e com sono de curta duração podem ter seu apetite aumentado devido à alteração de hormônios relacionados à saciedade e ao controle de peso. Ambos os casos estão associados com o aumento da ativação do sistema inflamatório. Embora a maioria dos estudos que investigaram os efeitos dos distúrbios do sono tenha sido realizada em indivíduos obesos, Kim et al. [23] mostraram evidências do efeito dos distúrbios do sono na presença 
de obesidade visceral (cintura abdominal aumentada) e de fatores de risco metabólicos em indivíduos não obesos.

Além dos fatores já citados, o tabagismo é um importante fator de risco modificável e tem associação epidemiológica com DCV em homens e mulheres. Em 2009, um estudo apurou que aproximadamente $50 \%$ dos pacientes de meia-idade, portadores de DCV, eram fumantes ou ex-fumantes [24]. As alterações vasculares, consequência do tabagismo, envolvem disfunção endotelial, anormalidades do metabolismo lipídico, alterações da coagulação sanguínea, oxidação e inflamação [22]. Nesse quesito, a amostra também mostrou níveis elevados de PCR, que está associada não somente à $\mathrm{SM}$, mas, também, às $\mathrm{DCV}$ e doenças cerebrovasculares [24].

No mesmo estudo mencionado no parágrafo anterior, PCR $>0,3 \mathrm{mg} / \mathrm{dl}$ correlacionou-se significativamente com cintura abdominal aumentada, glicemia $>110 \mathrm{mg} / \mathrm{dl}$ e IMC $>30 \mathrm{~kg} / \mathrm{m}^{2}$, sendo que a PCR foi o marcador inflamatório de maior expressão em relação às variáveis estudadas: tabagismo, albuminúria, história de cardiopatia prévia, IMC, cintura abdominal aumentada e hiperglicemia [24]. Assim, a PCR está associada às comorbidades da SM e fortemente relacionada às DCV [22,24]. Porém, quando se fala em qualidade de vida, também precisam ser analisados o perfil bioquímico e os níveis pressóricos do paciente, pois estes são outros indicadores da evolução e/ou do estado das comorbidades que acompanham a obesidade e fazem parte da SM [2,3]. Na amostra avaliada no presente estudo, a mediana de PAS foi de $140 \mathrm{mmHg}$, evidenciando o aumento do risco para DCV se levarmos em conta os resultados de triglicerídeos elevados. Qualquer um desses dois componentes da SM é fator de risco para as DCV, ao lado dos demais critérios de diagnóstico da SM definido pelo IDF [4].
A HAS essencial está associada, frequentemente, a diversas anormalidades metabólicas, sendo a obesidade, a intolerância à glicose e a dislipidemia as mais comuns. Também há evidências de que a resistência à insulina e a hiperinsulinemia resultem em aumento da reabsorção de sódio, aumentando o débito cardíaco e levando à vasoconstrição e, consequentemente, à HAS [25]. Assim, hipertrigliceridemia, resistência à insulina e LDL-c elevado podem promover a formação de ateromas. Essas alterações estão associadas ao estresse oxidativo e à disfunção endotelial, demonstrando a natureza pró-inflamatória da doença aterosclerótica macrovascular, bem como da própria SM e da obesidade [26,27].

O presente estudo apresenta algumas limitações. A principal refere-se à coleta de dados de prontuários médicos, que, por terem sido feitas por vários profissionais, não foram uniformes. Outra limitação é a não periodicidade das consultas pré-cirúrgicas, realizadas em diferentes datas e com diferentes profissionais das especialidades médicas e não médicas da equipe multidisciplinar, com prontuários não padronizados, pois atendem às especificidades das áreas.

Apesar dessas limitações, o estudo pôde demonstrar a complexidade do perfil do paciente obeso e portador de SM da amostra investigada. Grande parte era composta de mulheres adultas jovens, casadas, com escolaridade de nível superior e com a presença de várias comorbidades somadas ao excesso de peso. Os pacientes também apresentavam alterações de sono e altos níveis de PCR, referiam excesso de peso na infância e faziam uso de mais de um tipo de medicamento. Conhecer o perfil do paciente candidato à cirurgia bariátrica ajuda a orientar as ações da equipe envolvida e é fundamental para o sucesso do procedimento e para o seguimento posterior do paciente.

\section{NOTA}

Declaração de conflitos de interesse

Os autores declaram não haver conflitos de interesse relevantes ao conteúdo deste estudo.

\section{REFERÊNCIAS}

1. World Health Organization. Health topics. Obesity [Internet]. Copenhagen: WHO; 2015 [updated 2015 Dec 16; cited 2015 Dec 28]. Available from: http://www.who.int/topics/obesity/en/

2. Neff KJ, Olbers T, le Roux CW. Bariatric surgery: the challenges with candidate selection, individualizing treatment and clinical outcomes. BMC Med. 2013 Jan 10;11:8. http://dx.doi.org/10.1186/1741-7015-11-8

3. Alberti KG, Zimmet P, Shaw J; IDF Epidemiology Task Force Consensus Group. The metabolic syndrome - a new worldwide definition. Lancet. 2005 Sep 24-30;366(9491):1059-62. http://dx.doi.org/10.1016/S0140-6736(05)67402-8

4. International Diabetes Federation. Consensus worldwide definition of the metabolic syndrome [Internet]. Brussels: IDF; 2015 [updated 2015 Dec 17; cited 2015 Dec 22]. Available from: http://www.idf.org/metabolic-syndrome 
5. Finelli C, Padula MC, Martelli G, Tarantino G. Could the improvement of obesity-related co-morbidities depend on modified gut hormones secretion? World J Gastroenterol. 2014 Nov 28;20(44):16649-64. http://dx.doi.org/10.3748/wjg.v20.i44.16649

6. Reis CEG, Vasconcelos IAL, Barros JFN. Políticas públicas de nutrição para controle da obesidade infantil. Rev Paul Pediatr. 2011;29(4):625-33.

7. Kelles SMB, Diniz MFHS, Machado CJ, Barreto SM. The profile of patients undergoing bariatric surgery in the Brazilian Unified National Health System: a systematic review. Cad Saude Publica. 2015 Aug;31(8):1587-601. http://dx.doi. org/10.1590/0102-311X00022714

8. Garrow JS, Webster J. Quetelet's index (W/H2) as a measure of fatness. Int J Obes. 1985;9(2):147-53.

9. World Health Organization. Collaborating Centre for Drug Statistic Methodology, Norwegian Institute of Public Health. The Anathomical Therapeutic Chemical Classification System [Internet]. Oslo: WHO; 2015 [updated 2015 Dec 16; cited 2015 Dec 28]. Available from: http://www.whocc.no/atcddd/

10. Oliveira APF, Malheiros CA, Santos AS, Jesus SR, Manuel J. Perfil de pacientes submetidos à cirurgia bariátrica atendidos em um hospital universitário do município de São Paulo. Saúde Colet. 2009;35(6):275-9.

11. Diniz MFHS, Passos VMA, Barreto SM, Diniz MTC, Linares DB, Mendes LN. Perfil de pacientes obesos classe III do sistema público de saúde submetidos à gastroplastia em "Y de ROUX", no Hospital das Clínicas da UFMG: altas co-morbidades e mortalidade hospitalar. Rev Med Minas Gerais. 2008;18(3):183-90.

12. Oliveira MS, Lima EFA, Leite FMC, Primo CC. Perfil do paciente obeso submetido à cirurgia bariátrica. Cogitare Enferm. 2013;18(1):90-4. http://dx.doi.org/10.5380/ce.v18i1.31312

13. Cavalcante RC. Análise comportamental de obesos mórbidos e de pacientes submetidos à cirurgia bariátrica [dissertação] [Recife]: Universidade Federal de Pernambuco; 2009. 110 p.

14. Garciacaballero M, Reyes-Ortiz A, Martínez-Moreno JM, Toval-Mata JA. Glycemic and lipid metabolic disorders in diabetic and non-diabetic patients bmi $<35$ or $>35$ before gastric bypass. Nutr Hosp. 2014 May 1;29(5):1095-102.

15. Lam DW, LeRoith D. Metabolic Syndrome [Internet]. Bethesda; 2015 [updated 2015 May 19; cited 2015 Nov 28]. Available from: http://www.ncbi.nlm.nih.gov/books/NBK278936/

16. Oh S, Oh S. Epidemiological and genome-wide association study of gastritis or gastric ulcer in korean populations. Genomics Inform. 2014 Sept;12(3):127-33. http://dx.doi.org/10.5808/GI.2014.12.3.127

17. Wree A, Kahraman A, Gerken G, Canbay, A. Obesity affects the liver - the link between adipocytes and hepatocytes. Digestion. 2011;83(1-2):124-33. http://dx.doi.org/10.1159/000318741

18. Gerhard GS, Benotti P, Wood GC, Chu X, Argyropoulos G, Petrick A, Strodel WE, Gabrielsen JD, Ibele A, Still CD, Kingsley $\mathrm{C}$, DiStefano J. Identification of novel clinical factors associated with hepatic fat accumulation in extreme obesity. J Obes. 2014;2014:368210. http://dx.doi.org/10.1155/2014/368210

19. Costa AJRB, Pinto SL. Transtorno da compulsão alimentar periódica e qualidade de vida de pacientes candidatos a cirurgia bariátrica. Arq Bras Cir Dig. 2015;28(Supl.1):52-5. http://dx.doi.org/10.1590/S0102-6720201500S100015

20. Bandeira VAC, Oliveira KR. Potenciais interações entre medicamentos usados na síndrome metabólica. Sci Med. 2014;24(2):156-64. http://dx.doi.org/10.15448/1980-6108.2014.2.16330

21. Sotolongo OG, Gámez AA, Sierra YH. Influencia del síndrome metabólico sobre la evolución de pacientes. Rev Cuba Med Mil. 2014;43(4).

22. Junqueira ASM, Romêo Filho LJM, Junqueira CLC. Avaliação do grau de inflamação vascular em pacientes com síndrome metabólica. Arq Bras Cardiol. 2009;93(3):360-6. http://dx.doi.org/10.1590/S0066-782X2009001000008

23. Kim NH, Lee SK, Eun CR, Seo JA, Kim SG, Choi KM, Baik SH, Choi DS, Yun CH, Kim NH, Shin C. Short sleep duration combined with obstructive sleep apnea is associated with visceral obesity in Korean adults. Sleep. 2013 May 1;36(5):723-9. http://dx.doi.org/10.5665/sleep.2636

24. Organización Mundial de La Salud. Obesidad y sobrepeso. Nota Descriptiva no 311 [Internet]. Copenhagen: OMS; 2015 [updated 2015 Jan; cited 2015 Nov 28]. Available from: http://www.who.int/mediacentre/factsheets/fs311/es/

25. Hruby A, Hu FB. The epidemiology of obesity: a big picture. Pharmacoeconomics. 2015 July;33(7):673-89. http://dx.doi org/10.1007/s40273-014-0243-x

26. Kaur J. A Comprehensive review on metabolic syndrome. Cardiol Res Pract. 2014;2014:943162. http://dx.doi. org/10.1155/2014/943162

27. Araújo TF, Guimarães DF, Gomes ET, Melo Luz JC, Spini VBMG. Síndrome metabólica - fatores de risco e aspectos fisiopatológicos. RBM Rev Bras Med. 2011;68(7-8):233-7. 